\title{
Diagnosis, prognosis and clinical treatment of refractory atypical chronic lymphocytic leukemia with multiple B cells: A case report.
}

\section{Man Chen}

Hebei Yanda Lu Daopei Hospital

Huipeng Sun

Beijing Ludaopei Hospital

Lina Zhang

Beijing Ludaopei Hospital

\section{Haiyan Gao}

Second Affiliated Hospital of Harbin Medical University

\section{Minjing Fu}

Beijing Ludaopei Hospital

\section{Ting Li}

Beijing Ludaopei Hospital

\section{Aixian Wang}

Hebei Yanda Lu Daopei Hospital

\section{Xueying Wu}

Hebei Yanda Lu Daopei Hospital

Junyi Zhen

Hebei Yanda Lu Daopei Hospital

\section{Meiwei Gong}

Hebei Yanda Lu Daopei Hospital

\section{Qing Du}

Hebei Yanda Lu Daopei Hospital

\section{Ping Wu}

Hebei Yanda Lu Daopei Hospital

\section{Tong Wang}

Hebei Yanda Lu Daopei Hospital

Hongxing Liu

Hebei Yanda Lu Daopei Hospital

Xiaoge Zhou

Beijing Ludaopei Hospital

Hui Wang ( $\square$ ldpwanghui@163.com ) 


\section{Case report}

Keywords: CLL/SLL, peripheral blood cell, histopathology, cytogenetics, immunophenotype

Posted Date: June 15th, 2021

DOl: https://doi.org/10.21203/rs.3.rs-621348/v1

License: (c) (i) This work is licensed under a Creative Commons Attribution 4.0 International License. Read Full License 


\section{Abstract}

Background: Chronic lymphocytic leukemia/small lymphocytic lymphoma (CLL/SLL) is the most prevalent adult leukemia, and its incidence continues to rise year after year. Rapid and precise diagnosis is an essential element in effective case management, however, the clinical diagnosis, treatment, and prognosis of CLL/SLL are not fully elucidated. Case presentation: we report the case of a 66-year-old man with atypical CLL/SLL. The white blood cell (WBC) count $(842.0 \times 109 / \mathrm{L})$, platelet count $(30.6 \times 109 / \mathrm{L})$, and abnormal lymphocytes were increased in peripheral blood. Flow cytometry showed $98.34 \%$ of nucleated cells were malignant monoclonal mature B cell. Peripheral blood smear found the leukocytes and lymphocytes with abnormal morphology were increased. Fluorescence in situ hybridization showed CCND1 (11q23)/IGH (14q32) and abnormal chromosome 12 were invisible, 91\%-93\% of interphase nuclei presented D13S319 and TP53, 17p13.1 loss. Histopathology analysis of bone marrow observed the proliferation centers with immunoblasts. Immunohistochemistry showed that bone marrow was positive for PAX-5, CD20, CD23, and CD5, negative for CD3, cyclinD1, and sox11, and partial positive for Ki67. The patient was diagnosed as CLL/SLL based on above clinical and laboratory findings. The patient was managed with oral $50 \mathrm{mg}$ Vinetoc, fluid replacement, hydration and alkalinization, and the symptoms were significantly relieved.

Conclusions: This report further expands the knowledge of clinical diagnosis and treatment of atypical CLL/SLL.

\section{Main Text}

Chronic lymphocytic leukemia/small lymphocytic lymphoma (CLL/SLL) is a highly heterogeneous clinical course, with an increasing incidence [1-4]. Therefore, rapid recognition and timely treatment are crucial in achieving optimal outcomes.

A 66-year-old man was admitted to our hospital because of two-month history of asthenia, night sweats, vomiting, and hepatosplenomegaly. Presenting white blood cell (WBC) showed 842.0×10\%/L, $91 \mathrm{~g} / \mathrm{L}$ hemoglobin, and $88 \times 10^{9} / \mathrm{L}$ platelet count. Microscopic examination of peripheral blood smears observed abnormal lymphocytes was present in $95 \%$. The serum biochemical indices of the patient (Supplementary Table S1) showed $\beta 2$-microglobulin 5127.59 ug/L.

Peripheral blood smear (Fig 1A) showed that the abnormal leukocytes and lymphocytes were markedly increased. Approximately $93 \%$ of the cells were prolymphocytes (green arrow) with large cell bodies, round or irregular nuclei, ccumulated chromatin, obvious nucleoli. We also found $7 \%$ of immunoblast (yellow arrow) and small lymphocytes (red arrow, $<1 \%$ ). In the French American British classification [5], prolymphocytes $\geq 55 \%$ were considered as prolymphocytic leukemia (PLL), but morphological analysis observed the cell body of low proportion cells was smaller than that of CLL cells with normal lymphocytes. 
For precise diagnosis, we performed MICM diagnosis. Histopathology analysis (Fig 1B) of bone marrow observed large cells with median nucleoli (i.e., paraimmunoblast) in proliferation centers, small chronic lymphocytes with deeply stained in the periphery. Immunohistochemistry (Fig 1C) found that bone marrow was positive for PAX-5, CD20, CD23, and CD5, and partially Ki67(30\%), negative for CD3, cyclinD1, and sox 11 .

Flow cytometry (Fig 2A) of peripheral blood showed that $98.34 \%$ of nucleated cells was malignant monoclonal mature B cell with heterogeneous cell size. They expressed CD19, CD5, kappa bri, CD20, CD79b bri, CD25, slgM bri, CD200, CD23, CD11c, and CD49d, and partially FMC7. PLL cells usually has CD5- CD23- FMC7+ immunophenotype, which is different from CLL cells. CLL can't transform into PLL, and the increase of precursor cell only indicates the increased progression of CLL, which often accompanied by NOTCH1 or TP53 aberration [6]. Therefore, although prolymphocytes accounted for $93 \%$ in this patient, the possibility of PLL was still excluded. The Royal Marsden Hospital (RMH) score is used for the diagnosis of CLL/SLL by flow cytometry [7]: CD5+, CD23+, FMC7-, CD79b dim or CD22dim, monoclonal kappa/lambda dim or slg dim. For this patient, the RMH score was 3-4, the atypical chronic lymphocytic, atypical mantle cell lymphoma, and other CD5 + small B-cell lymphomas cannot be excluded by 3-4 score and strong positive expression of CD200 [8, 9].

Fluorescence in situ hybridization (FISH) (Fig 2B-2E) revealed that the fusion genes of CCND1 (11q23)/IGH (14q32) were not found, chromosome 12 showed no abnormality, 91\% of interphase nuclei detected loss of D13S319, and 93\% of interphase nuclei detected loss of TP53, 17p13.1. Combination of negative expressing cyclinD1 and sox11 (Fig 2D) indicated the exclusion of mantle cell lymphoma.

It has been reported that the gene expression (unmutated immunoglobulin heavy-chain variable gene, CD38, CD49d, ZAP-70), trisomy 12 abnormalities, 11q-, 13q-, 6q-, 17p- or TP53 mutations, and serum $\beta 2$ microglobulin deposition are associated with CLL progression and poor prognosis [10]. In this case, this patient expressed CD49d, which is a poor predictor of CLL and involved in tumor invasion [11]. In this patient, including D13S319 loss, TP53 gene loss, higher Ki67 positive rate, significant myeloproliferative centers, and cell transformation, and $\beta 2$-microglobulin up to $5127.59 \mathrm{ug} / \mathrm{L}$,were observed as factors indicating poor prognosis.

The patient was diagnosed with CLL/SLL (BinetC, RailV; IPI7 polarization) and initially managed with BR regimen (bendamustine $90 \mathrm{mg} / \mathrm{m} 2$ combined with Mabthera $375 \mathrm{mg} / \mathrm{m} 2$ ), but this therapy was ineffective. Then, he was treated with oral $50 \mathrm{mg}$ Vinetoc, and fluid replacement, hydration and alkalinization were performed to monitor changes in the condition. After 5 days, the patient had no fever, nausea, vomiting or other discomfort. His general state is good, aphemia, spleen was reduced and no palpable in pleura; WBC, $51.0 \times 10^{9} / \mathrm{L}$. After 15 days, the WBC decreased to $25.0 \times 10^{9} / \mathrm{L}$, the symptoms were significantly relieved.

In conclusion,this study elaborated the diagnosis, prognosis, and clinical standardized treatment of CLL/SLL, and illustrated the significance and value of complete MICM diagnosis. 


\section{Abbreviations}

CLL/SLL: Chronic lymphocytic leukemia/small lymphocytic lymphoma; WBC: white blood cell; FISH: Fluorescence in situ hybridization; CBC: complete blood count; PLL: prolymphocytic leukemia; RMH: Royal Marsden Hospital.

\section{Declarations}

\section{Acknowledgements}

Not applicable.

\section{Authors' Contributions}

Hui Wang, corresponding author, designed the research and revise paper. Xiaoge Zhou, Ping Wu,Tong Wang, Hongxing Liu, revise paper. Man Chen, First Author, Statistical Analysis, analyzed data and wrote the paper. Huipeng Sun, Lina Zhang, Minjing Fu and Haiyan Gao gather clinical data. Aixian Wang, Xueying Wu, Junyi Zhen, Meiwei Gong, Qing Du, test samples and report results.

\section{Funding}

No funding

\section{Availability of data and materials}

The data and materials used and analyzed during the current study are available from the corresponding authors on reasonable request.

\section{Declarations}

\section{Ethics approval and consent to participates}

The procedures reported in this manuscript were approved by the Ethics Committee of the General Hospital of Chinese People's Liberation Army and conducted in accordance with the Declaration of Helsinki. Written informed consent was obtained from each participant prior to specimen collection.

\section{Consent for publication}

Not applicable.

\section{Competing interests}

The authors declare no competing interest.

\section{Author details}


${ }^{1}$ Department of laboratory medicine,Hebei Yanda Ludaopei Hospital ,065201, Langfang, China

2Department of Pathology,Beijing Ludaopei Hospital ,100176, Beijing, China

${ }^{3}$ Department of laboratory medicine, Second Affiliated Hospital of Harbin Medical University, Harbin, China

\section{References}

1. Trimech, M., A. Letourneau, E. Missiaglia, B. De Prijck, M. Nagy-Hulliger, J. Somja, et al. Angioimmunoblastic T-Cell Lymphoma and Chronic Lymphocytic Leukemia/Small Lymphocytic Lymphoma: A Novel Form of Composite Lymphoma Potentially Mimicking Richter Syndrome. AM J SURG PATHOL. 2021.

2. Nadeu, F., J. Delgado, C. Royo, T. Baumann, T. Stankovic, M. Pinyol, et al. Clinical impact of clonal and subclonal TP53, SF3B1, BIRC3, NOTCH1, and ATM mutations in chronic lymphocytic leukemia. Blood. 2016; 127(17): p. 2122-2130.

3. Szoltysek, K., C. Ciardullo, P. Zhou, A. Walaszczyk, E. Willmore, V. Rand, et al. DAP Kinase-Related Apoptosis-Inducing Protein Kinase 2 (DRAK2) Is a Key Regulator and Molecular Marker in Chronic Lymphocytic Leukemia. INT J MOL SCI, 2020; 21(20): p. 7663.

4. Kapoor, I., J. Bodo, B.T. Hill, E.D. Hsi, and A. Almasan. Targeting BCL-2 in B-cell malignancies and overcoming therapeutic resistance. Cell Death Dis. 2020; 11(11): p. 941-941

5. Hallek, M., B.D. Cheson, D. Catovsky, F. Caligaris-Cappio, G. Dighiero, H. Döhner, et al. iwCLL guidelines for diagnosis, indications for treatment, response assessment, and supportive management of CLL. Blood. 2018; 131(25): p. 2745-2760.

6. Oscier, D., M. Else, E. Matutes, R. Morilla, J.C. Strefford, and D. Catovsky. The morphology of CLL revisited: the clinical significance of prolymphocytes and correlations with prognostic/molecular markers in the LRF CLL4 trial. Br. J. Haematol. 2016; 174(5): p. 767-75.

7. Quinquenel, A., T. Aurran-Schleinitz, A. Clavert, F. Cymbalista, C. Dartigeas, F. Davi, et al. Diagnosis and Treatment of Chronic Lymphocytic Leukemia: Recommendations of the French CLL Study Group (FILO). Hemasphere. 2020; 4(5): p. e473.

8. Matutes, E., K. Owusu-Ankomah, R. Morilla, J. Garcia Marco, A. Houlihan, T.H. Que, et al. The immunological profile of B-cell disorders and proposal of a scoring system for the diagnosis of CLL. Leukemia. 1994; 8(10): p. 1640-5.

9. Ting, Y.S., S. Smith, D.A. Brown, A.J. Dodds, K.C. Fay, D.D.F. Ma, et al. CD200 is a useful diagnostic marker for identifying atypical chronic lymphocytic leukemia by flow cytometry. Int $J$ Lab 
Hematol. 2018; 40(5): p. 533-539.

10. Oscier, D.G., A.C. Gardiner, S.J. Mould, S. Glide, Z.A. Davis, R.E. Ibbotson, et al. Multivariate analysis of prognostic factors in CLL: clinical stage, IGVH gene mutational status, and loss or mutation of the p53 gene are independent prognostic factors. Blood. 2002; 100(4): p. 1177-84.

11. Jain, N. New developments in Richter syndrome. Clin. Adv. Hematol. Oncol. 2015; 13(4): p. $220-2$.

\section{Figures}

A

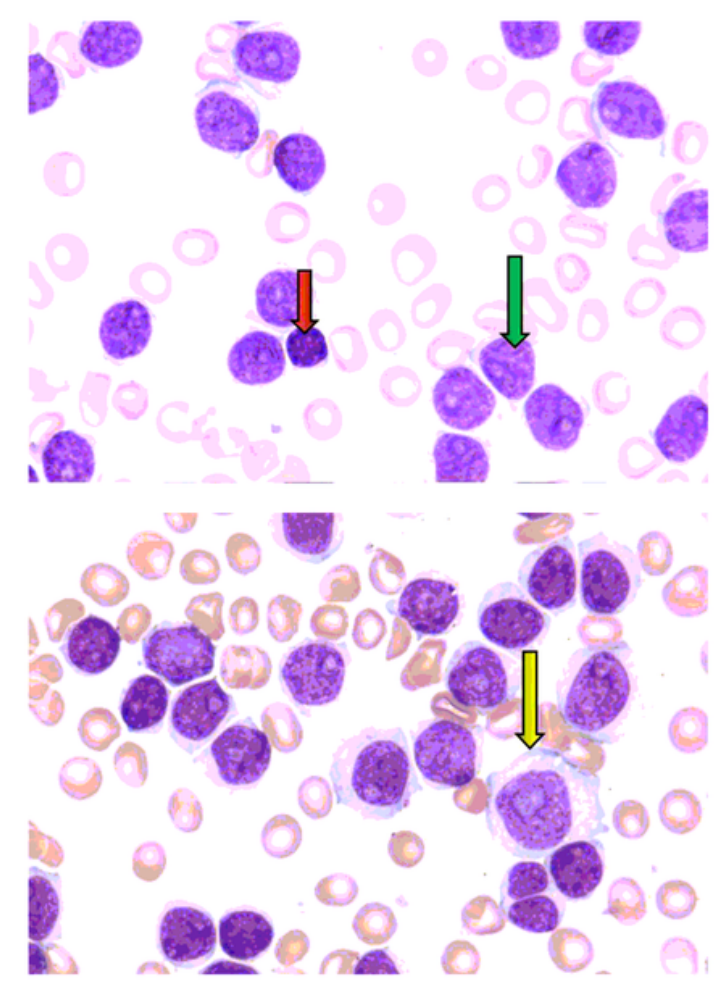

B

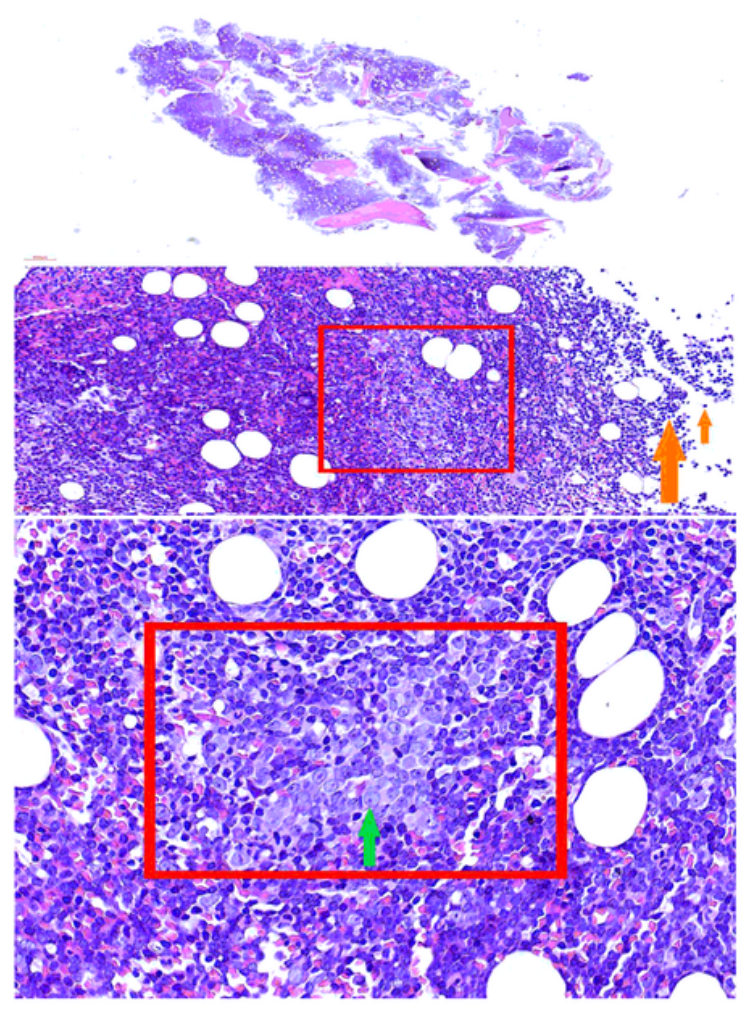

D

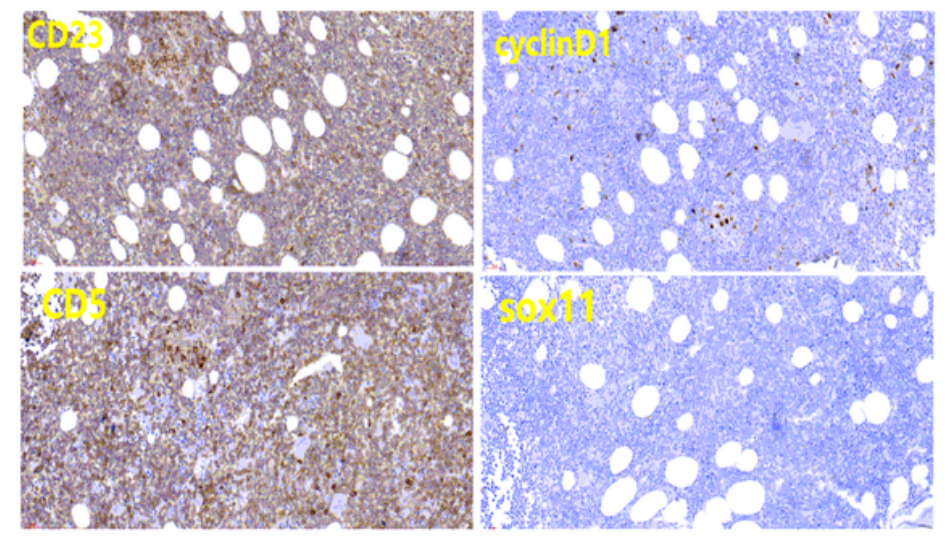

\section{Figure 1}


Analysis of peripheral blood and histopathology. (A) Peripheral blood was stained by Wright. (B) H\&E staining in bone marrow. Red box, proliferation center; green arrow, immunoblast; orange arrow, chronic lymphocyte. (C) Immunohistochemistry analysis for PAX-5, CD20, CD23, CD5, CD3, cyclinD1, sox11, and ki67 expression in bone marrow.

A
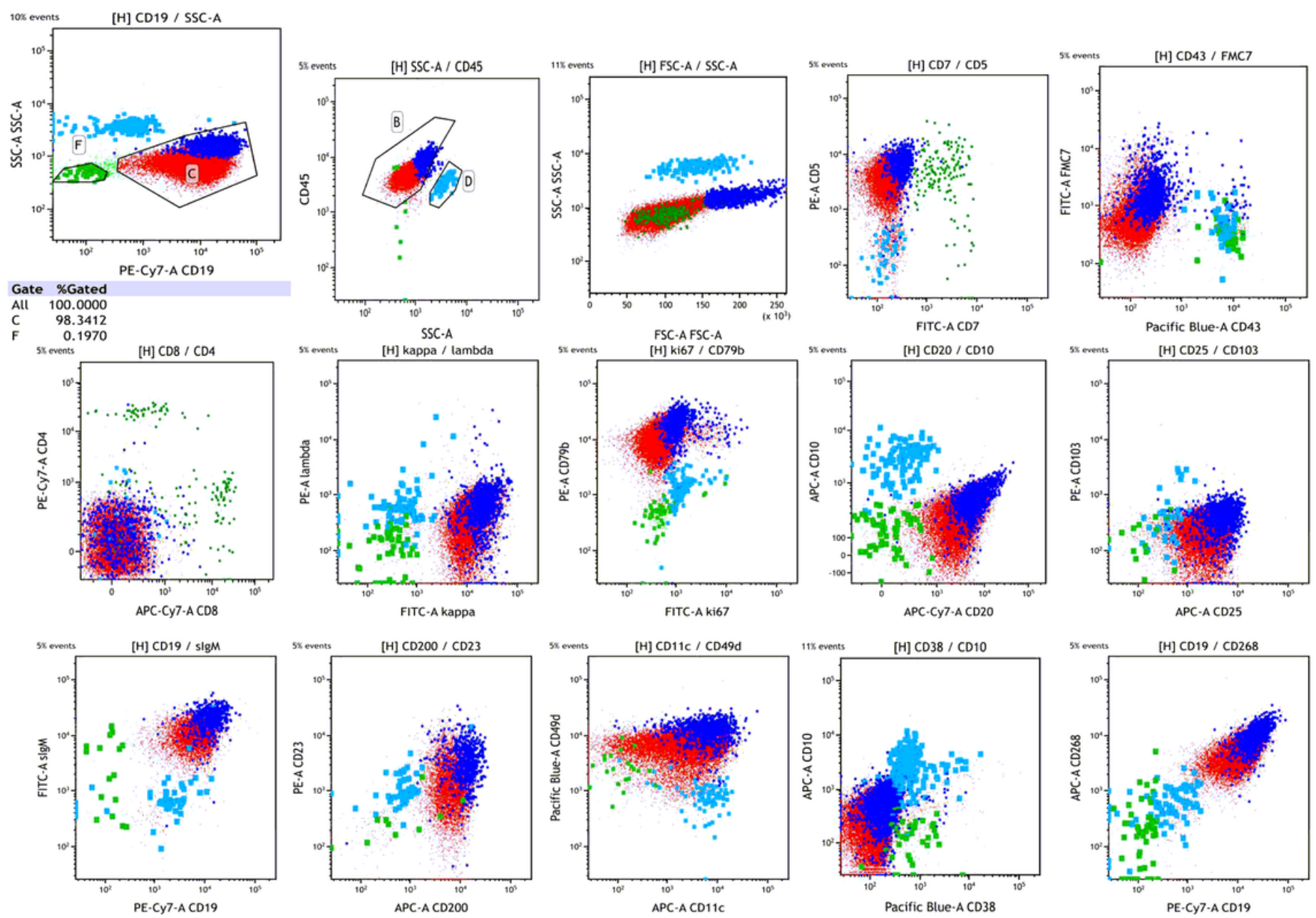

B

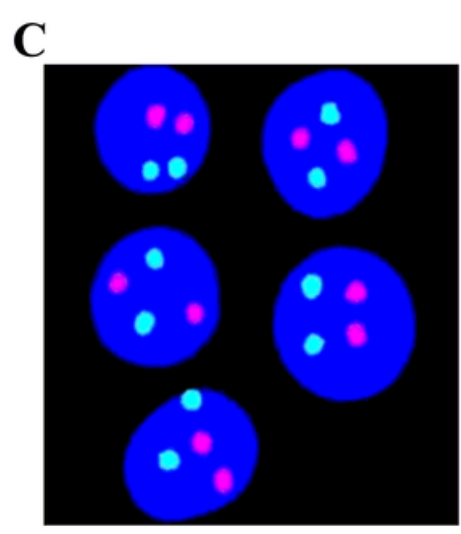

D

$\mathbf{E}$
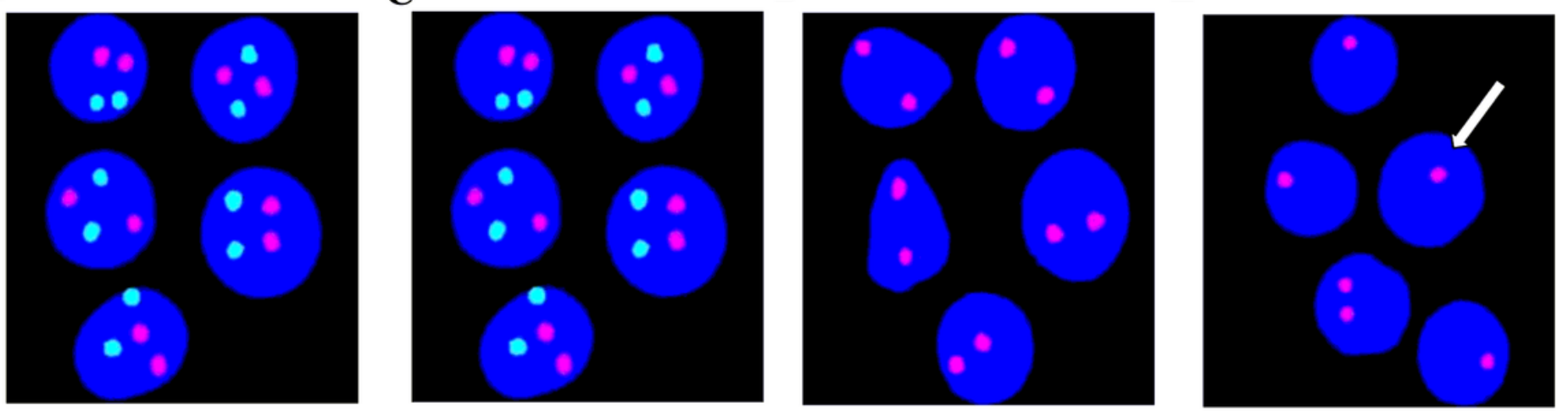

Figure 2

Analysis of immunophenotype and cytogenetics. (A) The immunophenotype of cells in the peripheral blood was analyzed by flow cytometry. (B) FISH analysis showing the $t(11 ; 14)$ (q13; q32) translocation. 
Green signal is CCND1, red signal is IGH. (C) The red probe detects the centromere of chromosome 12. (D) FISH detected loss of D13S319. Red signal and white arrow indicates del(13q14). (E) Detection of TP53/CEP17 site. Red signal is TP53, green signal is CEP17, white arrow indicates the loss of 17p13.1 and TP53.

\section{Supplementary Files}

This is a list of supplementary files associated with this preprint. Click to download.

- TableS1.doc 